\title{
Les grands systèmes botaniques depuis Linné
}

\author{
A propos du $250^{e}$ anniversaire de la naissance de Linné et de Buffon \\ Par Charles Baehni
}

En simplifiant un peu les choses, on peut dire que LinNé fut le dernier représentant marquant de la lignée des créateurs de systèmes artificiels. Après lui, il y aura des applications et des développements, mais la source de ceux qui ont si longtemps essayé de découvrir l'ordre dans la nature en considérant les variations d'un seul organe sera tarie: les CÉSALPIN (1519), Morison (1620), Ray (1628), Knauth (1638), Rivinus (1652), Tournefort (1656), Boerhaave (1668), Dillenius (1687), pour ne nommer que les plus importants, n'auront point de successeurs.

Il est cependant extrêmement difficile de se rendre compte, à l'heure actuelle, si Linné savait à quel point son système sexuel se plaçait dans la même perspective que les travaux de ses prédécesseurs. Lui-même était convaincu d'avoir innové; pour nous modernes, son système ne nous apparaît plus que sous la forme d'une des nombreuses méthodes artificielles, celle-ci développée, il est vrai, avec un talent incomparable, un souci de respecter les découvertes antérieures et surtout avec une sorte de pressentiment de l'importance qu'allait avoir la nomenclature binaire pour le développement de toutes les branches de la botanique ${ }^{1}$. Cette difficulté, nous l'éprouvons surtout parce que nous sommes habitués à penser en termes de mouvement, c'est-à-dire d'évolution et que nous ne pouvons pas facilement imaginer comment Linné concevait un système naturel qui ne fût pas en même temps phylétique. En effet, à part quelques exceptions dont il sera question plus loin, les botanistes du $\mathrm{XX}^{\mathrm{e}}$ siècle ne peuvent guère se représenter le monde végétal que sous la forme, sans doute simpliste et fausse dans son essence ${ }^{2}$, d'un arbre généalogique dont les racines plongeraient dans la matière inanimée des temps pré-primaires, dont les branches mortes figureraient les taxa éteints et dont les ultimes rameaux représenteraient les entités les

${ }^{1}$ Procédé qu'il n'a jamais revendiqué comme sien, mais qu'il a simplement étendu à toutes les espèces.

${ }^{2}$ Fausse pour plusieurs raisons, dont la plus apparente est que, si nous sommes assez bien renseignés sur l'apparition de petites formes nouvelles par mutation, nous sommes aussi peu avancés que LiNNÉ ou même que LAMARCK dans le domaine de la création des espèces, à plus forte raison, dans celle des genres et des familles. 
plus évoluées, les dernières créées. Un système naturel est donc pour nous l'image abrégée de créations successives échelonnées dans le temps. Ordinairement, on ne croît plus guère à l'apparition d'êtres organisés ex nihilo, mais nous devons nous rappeler toujours que cette hypothèse, condamnée par les meilleurs esprits, ne peut être ni démontrée ni infirmée. Linné, dont les allusions au système naturel sont assez nombreuses, envisageait l'ensemble des êtres organisés, la nature vivante, comme un puzzle dont il s'agissait de rapprocher les fragments. Ainsi, au numéro 77 de sa Philosophia botanica $^{3}$, il résume sa pensée: «Toutes les plantes se touchent entre elles comme les régions d'une mappemonde».

Voici bien longtemps que $\operatorname{Roscos}^{4}$, cherchant à défendre le système de Linné contre celui de Jussieu, démontrait d'une manière décisive que Linné croyait que les deux systèmes, le naturel et l'artificiel, pouvaient parfaitement coexister, mais avec cette restriction que le premier n'avait aucun intérêt pratique: mettre de l'ordre, un ordre humain certes, dans l'œuvre de la Nature, reconnaître les espèces, puis les genres, les définir les uns par rapport aux autres, telle serait, d'après Roscoe, la tâche du systématicien. En cours d'ouvrage, s'il observait quelques-uns de ces fils arachnéens qui lient entre eux les groupes naturels, il les signalerait à l'exemple de Linné qui en avait lui-même décelé un certain nombre. Mais quand le système serait complet, il se présenterait comme une mappemonde et son intérêt ne pouvait être que purement académique, puisque, quand on connaîtrait tous les genres et toutes les espèces (et Linné savait qu'à son époque on était loin de compte) les unités disposées naturellement s'engrèneraient les unes dans les autres, sans articulations, sans limites définissables; elles formeraient un tout si merveilleusement continu qu'aucun hiatus, aucune césure n'apparaîtrait. En d'autres termes, aucune classification ne serait plus possible, mais l'homme aurait retrouvé, non pour son usage personnel, mais pour le repos de son esprit, le plan secret de Dieu.

Ainsi, au fur et à mesure des découvertes, le système artificiel se serait étendu par remplissage des cases encore vides ${ }^{5}$ tandis que le système naturel,

${ }^{3}$ Linné, Philosophie botanique, traduction A. Quesné, Paris et Rouen 1788.

${ }^{4}$ Roscoe, "On Artificial and Natural Arrangements», in Transact. Linn. Soc. London. 11 (1815) 50.

5 A rapprocher du passage qu'on trouve dans la Philosophie botanique tout à la fin du $\mathrm{n}^{\circ} 77$ : Les plantes qui ne sont point encore connues rendent défectueuse la Méthode naturelle (c'est-à-dire le Système naturel, selon la terminologie actuelle): leur connaissance la perfectionnera, parce qu'en effet la Nature ne fait pas de sault. 
dont la recherche était recommandée aux botanistes entre autres, par la phrase bien connue: C'est le premier et le dernier vou des botanistes ${ }^{6}$ ne pouvait servir à quoique ce fût, si ce n'est à reconnaître la perfection de l'œuvre divine, dès l'instant qu'il serait achevé.

L'étude attentive des écrits de Linné ${ }^{7}$ nous a conduit à estimer la conclusion de Roscoe comme bien fondée. On remarquera qu'elle oblige à situer Linné en dehors du grand courant qu'on verra, tout à l'heure, partir de Ray et aboutir aux systèmes naturels actuellement en usage.

Les botanistes qui ont succédé à Linné n'ont guère fait qu'adopter le système sexuel en y insérant les nouveaux genres et nouvelles espèces que l'exploration des pays lointains leur faisait connaître: les noms de LiNNÉ fils, Houttuyn, Gronovius, Gmelin, Gilibert, Reichard, Murray, Persoon, Willdenow, Sprengel, enfin ceux de Roemer et Schultess appartiennent aux derniers botanistes qui se sont attachés à mettre à jour, selon un ordre artificiel, les connaissances des botanistes classificateurs.

On a voulu voir en Bernard de Jussieu (1699-1776) le père de la botanique systématique moderne, celui qui aurait découvert la méthode dite naturelle ${ }^{8}$. On sait maintenant que des rivalités mesquines sont à l'origine de cette désignation arbitraire et l'on s'étonne que des historiens sérieux comme $\mathrm{SACHS}^{9}{ }^{\text {ou comme MöBIUS }}{ }^{10}$ aient contribué à perpétuer cette erreur : la réalité est beaucoup moins simple; elle ne saurait plus nous apparaître aujourd'hui commé une sorte de génération spontanée qui se serait faite, tout d'un coup, sans avertissement et sans préparation, en la personne de l'honnête Bernard de Jussieu.

Si l'on dispose chronologiquement les noms des botanistes qui se sont attachés à créer, puis à perfectionner la méthode naturelle, on aperçoit immédiatement de nombreuses et inexplicables lacunes. On ne voit pas, en particulier, grâce à quel éclairage nouveau, quel brusque revirement, la théorie du système naturel, présentée au milieu du XVIII ${ }^{\mathrm{e}}$ siècle comme une sorte

${ }^{6}$ Philosophie botanique, $\mathrm{n}^{\circ} 77$.

${ }^{7}$ Philosophie botanique, $\mathrm{n}^{\circ} 77$ : Les fragments de la méthode naturelle doivent être recherchés avec le plus grand soin. Ibid., $\mathrm{n}^{\circ}$ 163: La méthode naturelle est le dernier terme de la botanique. Ibid., $\mathrm{n}^{\circ}$ 266: Plus les classes sont naturelles mieux elles valent, toutes choses égales. Voir aussi: Linné, Classes Plantarum. 1738. Praefatio et p. 484.

${ }^{8}$ Expression malheureuse en raison de la juxtaposition des deux mots méthode et naturelle, mais qui s'est perpétuée depuis le milieu du $\mathrm{XVIII}^{\mathrm{e}}$ siècle et qu'on ne saurait plus changer.

9 J. von SAchs, Geschichte der Botanik, traduit par H. DE VARIGNY, Paris 1892.

10 M. Möвıus, Geschichte der Botanik, Jena 1937. 
de jeu - difficile certes, mais peu utile - se trouva être, moins de 50 ans plus tard, démontrée avec assez d'évidence pour que tout le monde l'admît comme seule capable de faire progresser la science et cherchât à l'appliquer. Les botanistes se sont-ils avisés soudainement, avant même d'avoir épuisé les possibilités de son système, que Linné conduisait la botanique dans une voie sans issue? Que restait-il des grands efforts de RAY, de Tournefort, de Haller, dont les travaux semblaient brusquement ruinés par les succès de l'école suédoise ? N'existait-il pas, en dehors des idées généralement reçues un "parti de la résistance» qui aurait poursuivi les recherches dans une autre direction que les disciples linnéens ? De Ray à Magnol et de Magnol à BERNARD DE Jussieu, on suit mal l'enchaînement, et d'ailleurs presque toutes les phases du développement de la botanique au cours du XVIII ${ }^{\mathrm{e}}$ siècle semblent si mal se succéder qu'on est tenté de penser qu'il faudrait rajouter des pièces manquant au dossier pour qu'enfin apparaisse la continuité que l'histoire a sûrement dû présenter.

Or, Tournefort, Bernard de Jussieu, Adanson, A.-L. de Jussiev, Lamarck, A.-P. de Candolle ont appartenu au Jardin du Roy (devenu le Muséum d'Histoire Naturelle) ou bien ont appris leur métier dans cet établissement célèbre. Il convenait donc d'examiner s'il s'y rencontrait des circonstances favorables au développement de la systématique naturelle. A la suite de cette recherche, il s'est révélé que l'accord qui règne dans cette maison entre les diverses branches des sciences naturelles et singulièrement entre la zoologie et la botanique $a$, sinon déterminé la création du système naturel, tout au moins grandement facilité son éclosion.

C'est cette corrélation, ou mieux encore, cette interpénétration des deux disciplines, zoologie et botanique, qu'il ne paraît pas qu'on ait aperçue jusqu'aujourd'hui mais qu'on peut démontrer: les botanistes n'ont pas inventé seuls la méthode naturelle; ils ont fait cette découverte capitale en liaison avec les zoologistes qui paraissent en avoir eu les premiers l'idée.

Ces faits établis - et ils vont être étayés tout à l'heure - il convient de relire la Théorie élémentaire de la botanique d'A.-P. DE CANdolle pour y retrouver deux passages qu'il faut connaître pour comprendre comment les choses se sont passées. Page 84, on lit ceci: La classification naturelle du règne animal a été plus aisée et plus certaine que celle du règne végétal... et, page 59, cet autre passage: Que penserait-[on] d'un auteur qui, pour faire un livre élémentaire de zoologie, classerait les animaux d'après le nombre de leurs pattes; mettrait les serpens et les vers dans les apodes; les mollusques et les polypes dans les êtres qui n'ont qu'un pied; l'homme, la chauve-souris et 
les oiseaux dans la classe des bipèdes; les quadrupèdes ovipares et vivipares dans une quatrième, etc.?[On] dirait, sans doute, que cette manière de considérer les animaux peut bien avoir quelque commodité pour trouver les noms, ou quelque utilité dans l'étude des mouvements des animaux; mais que ce n'est pas la science elle-même, et qu'il faut bien se garder de suivre un ordre pareil dans l'étude. Pourquoi donc la même idée que personne n'a osé ni proposer, ni soutenir quant aux animaux, est-elle défendue avec tant de chaleur, quand il s'agit des plantes? Arrêtons là cette citation qui éclaire brusquement ce que Candolle semble avoir toujours su, mais que nous redécouvrons après lui, à savoir que les zoologistes étaient déjà bien engagés sur la voie de la classification naturelle au moment où les botanistes se sont avisés de les suivre.

En effet, les systèmes zoologiques naturels plongent leurs racines dans un lointain passé: en fait ils remontent aux premières ébauches d'Aristote et, même en tenant compte de nombreux essais d'imiter pour des raisons pratiques les systèmes artificiels des botanistes, la classification zoologique ne cessa jamais de tendre vers l'établissement d'un système naturel ${ }^{11}$.

Or, RaY (1628-1704), zoologiste (plus loin nous le verrons botaniste), collaborateur et successeur de Willoughby et tous ceux qui l'ont suivi, avait déjà décrit, avant le milieu du XVIII ${ }^{\mathrm{e}}$ siècle, un grand nombre d'animaux groupés en unités naturelles. Ray lui-même connaissait les Quadrupèdes, les Oiseaux, les Reptiles, les Cétacés, les Poissons, les Mollusques, les Crustacés, les Testacés, les Insectes; Linné distingua les Mammifères vrais (en y comprenant les Cheiroptères et les Cétacés). Si nous entrons dans le détail, nous voyons que chez les Oiseaux, il reconnaissait six ordres: les Rapaces, les Grimpeurs, les Palmipèdes, les Echassiers, les Passereaux, les Gallinacés. Cette simple énumération fait bien apparaître le fait sur lequel il faut insister: les groupes qui viennent d'être nommés ne se séparaient pas les uns des autres par un seul caractère dont on aurait utilisé les variations pour opérer des coupures, mais bien par une somme de signes particuliers, différents d'un groupe à l'autre. Bien que beaucoup de ces groupes fussent encore hétérogènes et continssent des éléments qu'on en fera sortir plus tard, l'essentiel était déjà trouvé: c'étaient des unités naturelles.

Cependant, ni Ray, ni Linné, n'avaient donné la théorie de la classification naturelle des animaux; ils avaient délimité les unités par instinct ou,

${ }^{11}$ E. GuYÉnot, Les sciences de la vie aux XVII et XVIII e siècles. L'idée d'évolution. Paris 1941. Voir p. 63. 
comme le dit A.-P. de Candolle, par tâtonnement. C'est à Buffon qu'on la doit et qui la publia en 1749, treize ans avant les Familles des Plantes par Adanson. On lit en effet, au premier volume de son Histoire naturelle, p. 21, ces lignes hautement significatives:... Presque tous les Nomenclateurs n'ont employé qu'une partie, comme les dens, les ongles ou ergots, pour ranger les animaux, au lieu de se servir de toutes les parties, et de chercher les différences ou les ressemblances dans l'individu tout entier: c'est renoncer volontairement au plus grand nombre des avantages que la nature nous offre pour la connaître que de refuser de se servir de toutes les parties des objets que nous considérons; et quand même on serait assuré de trouver dans quelques parties prises séparément, des caractères constants et invariables, il ne faudrait pas pour cela réduire la connaissance des productions naturelles à celles de ces parties constantes qui ne donnent que des idées particulières et très imparfaites du tout, et il me paraît que le seul moyen de faire une méthode instructive et naturelle, c'est de mettre ensemble les choses qui se ressemblent et de séparer celles qui diffèrent les unes des autres...

Voilà l'ordre méthodique qu'on doit suivre dans l'arrangement des productions naturelles; bien entendu que les ressemblances et les différences seront prises, non seulement d'une partie, mais du tout ensemble, et que cette méthode d'inspection se portera sur la forme, sur la grandeur, sur le port extérieur, sur les différentes parties, sur leur nombre, sur leur position, sur la substance même de la chose, et qu'on se servira de ces élément en petit ou en grand nombre, à mesure qu'on en aura besoin.

Ainsi, du côté des zoologistes, la situation est parfaitement nette: non seulement la plupart des grandes coupures naturelles sont reconnues, mais encore la théorie a été exposée par Buffon avec toute la clarté désirable ${ }^{12}$.

Du côté des botanistes, il n'en va pas de même. Ray ${ }^{13}$ (considéré maintenant comme botaniste) avait défini l'espèce. Après lui, Tournefort ${ }^{14}$ avait

${ }^{12}$ Il faut reconnaître que BufFon, cependant, ne tira pas la conséquence logique des idées qu'il avait si bien développées. Si la théorie du système naturel fut parfaitement exposée par lui-même, il ne l'appliqua pas. Dans la suite de la préface dont un extrait a été donné ci-dessus, il s'est efforcé de démontrer l'absurdité du système linnéen, mais sans parvenir à en donner un autre qui fût meilleur. Voir à ce sujet l'ouvrage capital de GuYÉNoT ${ }^{11}$ auquel nous avons fait de larges emprunts et dont on ne saurait se passer si l'on veut connaître l'évolution des idées au XVIII ${ }^{\mathrm{e}}$ siècle.

${ }^{13}$ J. RAY, Methodus plantarum nova, Amsterdam 1682; Historia Plantarum, Londres 1686. Voir en particulier lib. I, cap. XX.

14 Joseph Pitton de Tournefort, Elemens de botanique, Paris 1694; Institutiones rei herbariae, Paris 1700. 
caractérisé les genres, mais c'est à Magnol ${ }^{15}$ que revient le mérite d'avoir donné les premières descriptions des familles (que Tournefort ne semble pas avoir remarquées).

Nous trouvons dans sa préface la confirmation éclatante que ce grand pas vers un système naturel n'a pu être fait qu'après une étude approfondie des résultats déjà obtenus à cette époque par les zoologistes.

Voici les propres paroles de Magnol ${ }^{16}$ : Tandis que je pesais ces difficultés, il me vint à l'esprit d'examiner les genres des animaux et leurs différences pour voir si, partant de leurs caractères génériques, je pouvais également tirer des caractères pour les plantes. Je vis premièrement qu'il existait un très grand nombre de familles d'animaux que leurs caractères propres distinguaient, secondement, que dans ces mêmes familles se trouvaient des espèces variées; ainsi, parmi les bêtes, on compte de nombreuses espèces de chiens, parmi les oiseaux, de nombreuses espèces de colombes, et il existe encore parmi ces espèces, un assez grand nombre de degrés de parenté... Je m'aperçus que l'on retrouvait également dans les plantes tous ces degrés de parenté... Que Ray, à la fois zoologiste et botaniste, ait pu être conscient de l'existence de groupes naturels, ce fait n'a rien pour nous surprendre. Avec Magnol, nous assistons au phénomène décrit par A.-P. de Candolle, celui du botaniste se tournant délibérément vers le règne animal pour y puiser son inspiration.

Magnol avait eu à Montpellier comme élève Antorne de Jussieu qui, à l'âge de 22 ans, devait remplacer Tournefort. Adonné à la médecine plutôt qu'à la botanique, Antoine ne publia guère, mais il eut le mérite d'appeler auprès de lui son frère Bernard, lui aussi ancien élève de l'Ecole de Montpellier. Les leçons de Magnol ne furent donc pas perdues, ni celles de Buffon que l'on vient de citer. Bernard de Jussieu devait arriver au système naturel, mais tout occupé par ses leçons pour lesquelles il déployait un réel talent et par ses observations, il n'éprouva pas le besoin de publier le résultat auquel, dit-on, il parvint: la liste de 58 familles naturelles publiée tardivement par son neveu, Antoine-Laurent, et dont l'intérêt était déjà dépassé au moment où elle voyait le jour.

Mais Bernard avait formé Michel Adanson. Porté aux nues par les uns, décrié par les autres, Adanson s'est défendu d'avoir voulu publier sous son nom (comme on l'en a accusé) les découvertes de son maître. Il se défend même d'avoir su que Magnol, avant lui, avait déjà distingué des familles

15 P. Magnol, Prodromus historiae generalis plantarum in quo Familiae Plantarum per tabulas disponuntur, Montpellier 1689.

${ }^{16}$ P. Magnol, Prodromus, $3^{\mathrm{e}}$ page de la Préface, traduction. 
naturelles ${ }^{17}$. Néanmoins, il reconnaît honnêtement que le mérite de la découverte doit rester au vieux professeur montpelliérain. Il est donc vraisemblable qu'il a retrouvé tout seul (vers 1750), en confrontant la nature exotique avec les deux seuls livres qu'il avait avec lui pendant son séjour en Afrique, ceux de Tournefort et de Linné, la grande idée des groupes naturels, mais il est hors de doute aussi qu'il y avait été préparé par Bernard de Jussieu et que, lorsque rentré en France, il composait ses Familles des Plantes, il avait été puissamment aidé par la découverte d'idées semblables aux siennes dans les ouvrages de Buffon, qu'il cite d'ailleurs abondamment.

Antoine-Laurent de Jussieu, neveu de Bernard, a publié, comme il vient d'être dit, Ja liste des groupes naturels distingués par son oncle en tête de son Genera Plantarum secundum ordines naturales disposita (Paris 1789). Plus intéressé à ériger le monument familial qu'à rendre justice à ses prédécesseurs, Antoine-Laurent ne fait guère que saluer Adanson au passage, prenant soin de dater 1759 l'arrangement exécuté dans le parc du Trianon par Bernard de façon à faire bien comprendre qui de son oncle ou d'Adanson (publiant en 1763) était arrivé au but avant l'autre. Tout cela n'a pas d'importance: en vérité, il est indéniable qu'Antoine-Laurent, malgré ses petites roublardises, est le premier systématicien botaniste appliquant la méthode naturelle qui ait été entendu. Son ouvrage prenait la suite de Buffon et non celle d'Adanson; il paraissait en même temps que les travaux zoologiques de LACÉPÈDE, et précédait de peu ceux de GEOFFroy St-Hilaire et surtout de Cuvier, enfin il préparait la voie où Lamarck devait s'illustrer. Il n'a donc certainement pas inventé la méthode, mais il l'a brillamment utilisée et surtout, il a introduit dans la systématique un élément inconnu de ses prédécesseurs, la notion fondamentale de l'importance relative des caractères utilisés, celle de leur subordination.

On connaît les développements ultérieurs: la contribution géniale d'Augustin-Pyrame de Candolle, théoricien et praticien, celle de R. Brown, d'Endlicher, de Bentham et Hooker, d'Engler et Prantl, de Bessey, de Wettstein, de Pulle, de Hutchinson, de Gundersen. Les systèmes pourront différer par les conceptions philosophiques sur lesquelles ils seront fondés, ou simplement par le choix des caractères essentiels, ils pourront même être partiellement transformés par la découverte de faits nouveaux; la méthode ne changera plus, elle ne fera que se perfectionner.

17 Voir sur cette mise au point: A. Chevallier, Michel Adanson, voyageur, naturaliste et philosophe, Larose, Paris 1934, p. 113. 
La découverte du fait que les botanistes se sont appuyés sur leurs confrères zoologistes pour parvenir à la notion des groupes naturels pourrait peut-être chagriner les premiers. On veut croire, cependant, qu'il n'en sera rien, car ils savent eux aussi que la recherche historique ne peut pas avoir comme conclusion une distribution de médailles. Le but qu'elle se propose c'est de connaître le cheminement des idées et aucun cachet marquant le mérite ou le démérite ne doit être apposé sur les œuvres des hommes de science, tant que ceux-ci restent de bonne foi.

L'image qui s'est fixée dans l'esprit des botanistes, comme il a été rappelé au début, est celle d'un arbre généalogique. Au fur et à mesure que la paléontologie, la géobotanique, la morphologie comparée surtout, faisaient des progrès, enfin sous l'influence des théories de l'évolution de LAMarcK puis de DARwin, l'arbre changeait de forme; cependant c'était toujours un arbre.

L'étendard de la révolte contre cette manière statique de se représenter le monde végétal fut brandi par HALlier; dans une série de travaux ${ }^{18}$, il attira l'attention des botanistes sur la multiplicité presque infinie des ponts qui relient entre eux les groupes dits naturels. Cependant, incapable de rendre aisément accessibles ses idées bouillonnantes, il n'eut guère d'influence sur la marche de la science. Sa succession fut reprise par le botaniste japonais HaYata ${ }^{19}$ à qui l'on doit un étonnant système, appelé «dynamique» qui repose sur deux hypothèses: premièrement, que les espèces ancestrales étaient aussi nombreuses que les espèces actuelles; deuxièmement que les espèces ancestrales ainsi que les actuelles sont unies entre elles par des liens formant un réseau. Il est évident que la première supposition n'est pas démontrable, mais nous nous rappellerons cependant que l'idée couramment acceptée de la vie commençant sous l'apparence d'une monade ne l'est pas non plus. Quant à la seconde, elle répond si bien à ce que chacun a pu observer, à tous les échelons de la hiérarchie, qu'elle nous paraît parfaitement acceptable. Il faut, en suivant l'idée de Hayata, non pas considérer le monde vivant comme un réseau à deux dimensions, mais à 4,5 et

18 Voir spécialement H. HALlier, «Provisional scheme of the natural (phylogenetic) system of flowering plants», in New Phytol. 4 (1905) 151-62. Voir aussi: Die Grundlagen des Hallierschen Angiospermensystems. G. SENN, Beiheft zum Botanischen Centralblatt 17 (1954) 129.

19 B. Hayata, «The Natural Classification of Plants According to the Dynamic System», in Ic. Pl. Formos. 10 (1921) 97-234; "Über das ,Dynamische System“ der Pflanzen», in Ber. dtsch. Bot. Gesellschaft 49 (1931) 328. Voir à ce sujet: G. F. Du RıEtz, Svensk Bot. Tidskr. 24 (1930) 335. 
plus encore, selon le nombre de facteurs envisagés ou, comme le veut l'auteur, selon le nombre de gènes.

L'image que nous pouvons nous former des êtres vivants n'est donc pas celle d'un arbre généalogique, mais d'un réseau dont l'apparence change selon le temps et l'observateur, les nœuds (les points de condensation) représentant les unités taxonomiques. C'est l'ampleur de ces changements qui a déterminé la désignation de «système dynamique».

Il ne semble pas que les travaux de RoBerty aient eu comme point de départ la théorie de Hayata, mais il est certain que les deux auteurs, japonais et français, ont creusé le même sillon.

Roberty est certainement le dernier venu de cette courte série des nonconformistes. Son système n'a pas encore été publié, mais il le sera sous peu, en deux parties: une première consacrée à la théorie et une seconde, à un essai d'application dans la famille des Graminées.

Selon Roberty (et c'est ici qu'il rejoint et prolonge Hayata), les gènes se sont conduits, au cours de l'évolution, comme des unités indépendantes. Cependant, l'évolution étant irréversible, les apparents retours en arrière proviennent du fait qu'elle se meut en spirale et qu'éventuellement, deux tours de spire peuvent se superposer. Enfin, la différenciation s'opère par perte de potentialité, grâce à l'action du milieu sur la matière vivante. L'auteur détermine le choix des caractères significatifs non plus par une sélection arbitraire ou par instinct (le fameux flair des systématiciens), mais par une méthode mathématique où tous les caractères connus sont analysés de façon à faire apparaître les indices de corrélation. Les caractères étant ainsi mathématiquement choisis, tout le système se ramène à un schéma de présence-absence, avec, entre les deux pôles, une zone indifférenciée.

La famille (unité fondamentale) aura donc nécessairement 3 sousfamilles, chaque sous-famille 9 sous-tribus arrangées en 5 tribus, chaque sous-tribu se trouvera divisée en 81 genres, etc.

Le nombre des espèces possibles, dans chaque famille, sera finalement de 6561 multiplié par $81 \times 9 \times 3$ espèces. Il est évident qu'il n'en existe pas autant; mais le réseau est construit de telle façon qu'il comprend à côté des espèces actuelles, les espèces disparues, les espèces non viables, les espèces non encore découvertes et celles non encore créées.

Roberty, en somme, traite les caractères spécifiques, génériques et familiaux comme des unités géniques, de la même façon que les généticiens travaillant à l'échelle des formes et des variétés. L'essai d'application de cette théorie auquel l'auteur est en train de mettre la dernière touche dans 
la famille des Graminées (famille où chaque auteur a pu, à son aise, jusqu'aujourd'hui créer son propre arrangement, d'ailleurs aussitôt contredit) servira de test. L'idée est neuve d'appliquer des méthodes mathématiques ${ }^{20}$ indépendantes du jugement des botanistes, à l'élaboration d'un système assez vaste pour n'avoir pas besoin d'être remanié chaque fois qu'on trouvera un nouveau cas.

Deux siècles et demi se sont écoulés depuis la naissance de Linné. Nous sommes bien loin d'avoir découvert l'arrangement idéal dont l'auteur suédois soupçonnait l'existence. Est-ce à dire que nous en sommes au même point qu'en 1707? Buffon, déjà en 1749, a donné, par avance, réponse à cette question ${ }^{21}$ : Il semble que la recherche de cette méthode générale soit une espèce de pierre philosophale pour les Botanistes, qu'ils ont tous cherchée avec des peines et des travaux infinis... Il est arrivé en Botanique ce qui est arrivé en Chymie, c'est qu'en cherchant la pierre philosophale, que l'on n'a pas trouvée, on a trouvé une infinité de choses utiles; et de même, en voulant faire une méthode générale et parfaite en Botanique, on a plus étudié et mieux connu les plantes et leurs usages... Buffon jugeait une ère qui allait se terminer avec Linné et avec lui-même. A plus forte raison, ces remarques si justes sontelles valables à notre époque. L'abondante moisson des découvertes qui ont été rendues possibles grâce aux systèmes arbitraires et incomplets dont on a essayé de résumer l'histoire est la preuve que même si nos efforts ne devaient jamais nous donner la clef de l'ordre qui règne dans la nature, il est bon et nécessaire que ces efforts soient poursuivis.

20 Voir cependant Linné, Philosophie botanique, $\mathrm{n}^{\circ} 166$, et surtout OKEN, Esquisse du système d'anatomie, de physiologie et d'Histoire naturelle, Paris 1821; de son Lehrbuch der Naturgeschichte, seul le Zweyter Theil (Botanik), zweyte Abtheilung, erste Hälfte (Merk- und Stamm-Pflanzen) a été vu; voir aussi (selon Möвıus, p. 52) Allgemeine Naturgeschichte für alle Stände (Botanik), Band 2, 1851, où l'auteur calcule le nombre des classes possibles (256) dont chacune a 16 genres et chaque genre 16 espèces, de sorte que le règne végétal se composerait de 65536 espèces.

21 Buffon, Histoire naturelle, Vol. 1, 14 (1749). 